\title{
STUDY OF LARGE-SCALE BLOCK ADJUSTMENT WITH LASER ALTIMETRY DATA GLAS OF ZY3 IMAGES OVER SHANDONG
}

\author{
Taiping Wang ${ }^{1, *},{\mathrm{Xin} \mathrm{Li}^{1}}^{1}$ \\ ${ }^{1}$ School of Remote Sensing and Information Engineering, Wuhan University, Wuhan, 430079, China, \\ 1351755632@qq.com,xli2126@whu.edu.cn
}

KEY WORDS: ZY3, GLAS, Block Adjustment, Without Ground Control, Elevation accuracy

\begin{abstract}
:
In the global mapping work, there will be no control points or uneven distribution of control points. The traditional method of single scene image correction based on ground control points is not suitable for modern surveying and mapping, which has no available control points in a large range. New control data is urgently needed to make up for the influence of traditional control points on surveying and mapping. In this paper, aiming at improving the elevation accuracy of ZY-3 uncontrolled point surveying, a filtering principle based on the analysis based on waveform analysis and the stereo image pair based on tie points is proposed for the screening of GLAS laser elevation points. A new method using high-reliability laser elevation control points as the height constraint and the regional network adjustment of ZY-3 images without control points is studied. Through the experiment in Shandong Province, it is proved that the elevation accuracy of the adjustment can be improved obviously by introducing the selected laser points. The mean error of adjustment elevation is increased from -2.297 to $0.216 \mathrm{~m}$ and the mean square error of elevation is increased from $3.193 \mathrm{~m}$ to $2.007 \mathrm{~m}$, significantly improving the elevation positioning accuracy of adjustment.
\end{abstract}

\section{INTRODUCTION}

With the continuous development of science and technology, people can use optical satellites to quickly obtain massive image data covering the earth's surface in a short time. Using these data for regional network adjustment, we can obtain high-precision DOM and DSM of the earth's surface, and provide other industries with high-precision spatial reference data. Block adjustment is a key step in the process of map making. The quality of the adjustment results directly determines the geometric accuracy of the final product, which has been concerned by photogrammetry workers. In the actual mapping engineering, a certain number of ground control points should be evenly arranged in the survey area to ensure the geometric accuracy of the adjustment results (Zhang et al, 2009). However, in the process of performing block adjustment, it is inevitable to encounter some areas without ground control points (GCP) or with just a few ground control points, such as desert, forest, plateau and so on. In the case of largescale adjustment without ground control point, the degree of freedom of the adjustment model is high. Directly taking the parameters to be adjusted as the free unknowns will lead to the ill condition of the normal equation matrix, thus making the adjustment accuracy unstable and error prone to excessive accumulation, resulting in the distortion and deformation of the network ( $\mathrm{Li}, 1982)$. The traditional regional network adjustment method can not meet the requirements of high-precision adjustment in these areas. Therefore, the adjustment method without ground control of the stereo satellite image has been concerned and studied by many scholars.

With the development of satellite sensor manufacturing technology, orbit and attitude determination and sensor on-orbit calibration, the quality and initial positioning accuracy of satellite image have reached a high level, which provides the image data basis for the realization of block adjustment method without ground control. With the release of various high-resolution and highprecision DEM, DEM is taking as auxiliary data to adjust the real data obtained by ZY-3 three line array camera and GF-1 panchromatic multispectral sensor, and the plane accuracy can reach 0.5 pixels (Zheng et.al, 2016). The virtual control points of ZY-3 satellite image are generated by RPC model and introduced into adjustment model as weighted observation value, so that the positioning accuracy of ZY-3 image can reach $5 \mathrm{~m}$ (Yang et.al, 2017). In addition, using the highresolution satellite image with higher positioning accuracy as the control condition has also achieved better adjustment effect (Li et.al,2008).

In 2003, ICESat, the first earth to earth laser altimeter satellite, was launched in the United States. It was equipped with the geoscience laser altimeter system (GLAS), witch its elevation measurement accuracy can reach $10 \mathrm{~cm}$. By the time it stopped, it had collected hundreds of millions of high-quality laser altitudes. These data can cover the whole world, and its elevation measurement accuracy can be comparable with GPS data, and can be used as a new elevation data to assist

* Corresponding author 
the global mapping work. In the absence of field control points, the GLAS laser altimetry data screened by the public SRTM DEM can improve the elevation accuracy of the block adjustment of the mapping satellit-1 stereo image from the original $5.88 \mathrm{~m}$ to $2.51 \mathrm{~m}$, which verifies the effectiveness of ICESat laser points assisted block adjustment for mapping satellite1 stereo imagery (Wang et.al, 2018).

In this paper, the laser altimetry data obtained by ICESat is used as the elevation control data to carry out the selection of laser elevation control points and assist the optical stereo surveying satellite to carry out block adjustment. This paper proposes a laser data screening mechanism based on the precise screening of waveform data and the re-screening of stereoscopic image pairs of tie points, and GLAS laser altimetry data with high reliability is screened out. A comparative experiment is carried out in Shandong Province. One is the completely uncontrolled free network adjustment of ZY-3 images, the other is the GLAS laser altimetry data assisted block adjustment of ZY-3 images. By using high-precision field GPS checkpoints to make quantitative analysis of the experimental adjustment results, the feasibility of using GLAS laser altimetry data as elevation assistant to improve the elevation accuracy of block adjustment of ZY-3 images is verified.

\section{METHOD}

\subsection{Block adjustment based on laser altimeter data}

We use the RPC model to investigate the ZY-3 satellite image.

$$
\begin{aligned}
& r=F(\text { Lat }, \text { Long }, \text { Height }) \\
& c=G(\text { Lat }, \text { Long }, \text { Height })
\end{aligned}
$$

Where $(r, c)=$ image pixel coordinates.

After the on-orbit geometric calibration and the sensor correction, the error in the originally provided RPCs of the ZY-3 image is mainly in linear pattern (Cao, 2015). In order to further reduce the remaining error, we choose the affine transformation as the image-space additional mathematical model as shown in Eq. (2).

$$
\begin{aligned}
& \Delta r=a_{0}+a_{r} \cdot r+a_{c} \cdot c \\
& \Delta c=b_{0}+b_{r} \cdot r+b_{c} \cdot c
\end{aligned}
$$

Where $\Delta r, \Delta c=$ the correction of image pixel coordinates;

$$
a_{0}, a_{r}, a_{c}, b_{0}, b_{r}, b_{c}=\text { the }
$$

affine transformation parameters to be solved in adjustment.

According to the affine transformation and the RPC model, we can list the error function as shown in Eq. (3).

$$
\begin{aligned}
& v_{r}=F(\text { Lat }, \text { Long }, \text { Height })-\Delta r-r \\
& v_{c}=G(\text { Lat }, \text { Long, Height })-\Delta c-c
\end{aligned}
$$

Two error functions can be listed by using RPC model and coordinates of the image points. There are two types undetermined parameters, the object coordinates and the image space additional parameters. For the control points, the object coordinates are accurately known, these two error functions are linear. For the tie points, both undetermined parameters are unknown, these two error functions are nonlinear. We can linearize these two error functions by Taylor expansion. The matrix form of the linearized error functions are shown as Eq. (4).

$$
\left[\begin{array}{l}
v_{r} \\
v_{c}
\end{array}\right]=\left[\begin{array}{lll}
\frac{\partial r}{\partial \text { Lat }} & \frac{\partial r}{\partial L o n g} & \frac{\partial r}{\partial H e i g h t} \\
\frac{\partial c}{\partial \text { Lat }} & \frac{\partial c}{\partial \text { Long }} & \frac{\partial c}{\partial \text { Height }}
\end{array}\right] \cdot\left[\begin{array}{c}
\Delta \text { Lat } \\
\Delta \text { Long } \\
\Delta \text { Height }
\end{array}\right]-\left[\begin{array}{ccc}
1 & r c 000 \\
0001 & r c
\end{array}\right] \cdot\left[\begin{array}{l}
\Delta a_{0} \\
\Delta a_{r} \\
\Delta a_{c} \\
\Delta b_{0} \\
\Delta b_{r} \\
\Delta b_{c}
\end{array}\right]-\left[\begin{array}{c}
r \\
c
\end{array}\right]
$$

Since the laser points have higher height precision and lower plane precision, the elevation value of the laser points are taken as the elevation constraint condition. For the ZY-3 three-line array images, we select the tie points obtained by automatic matching within a certain range of the laser point, their object coordinates will be got by forward intersection of stereo image pair. Then we assign the elevation coordinates of the laser point to the tie point closest to the laser point, while the plane coordinates of the tie point remain unchanged. These tie points will be given higher weights, while general join points will have lower weights.

\subsection{Laser elevation point screening}

\subsubsection{Gaussian parameter estimation and background noise filtering}

For the standard gaussian function, its main parameters include gaussian amplitude $A_{m}$, mean value $t_{m}$ and standard deviation $\sigma_{m}$. By taking the first and second derivatives of the standard gaussian function and setting its value to 0 , the amplitude of the waveform and the position of the initial wave peak can be obtained.

For the gaussian function with only noise, its mathematical expression is shown in Eq. (5)( Brenner,2011).

$$
\omega_{m}=A_{m} e^{\frac{-\left(t-t_{m}\right)^{2}}{2 \sigma_{m}^{2}}}+\varepsilon
$$

The first and second partial derivatives with respect to parameters $t_{m}$ are:

$$
\frac{\partial \omega_{m}}{\partial t}=-A_{m} \frac{-\left(t-t_{m}\right)^{2}}{2 \sigma_{m}^{2}} e^{\frac{-\left(t-t_{m}\right)^{2}}{2 \sigma_{m}^{2}}}=-\omega_{m} \frac{\left(t-t_{m}\right)^{2}}{\sigma_{m}^{2}}
$$




$$
\frac{\partial^{2} \omega_{m}}{\partial t^{2}}=\omega_{m}\left[\frac{\left(t-t_{m}\right)^{2}}{\sigma_{m}^{2}}-\frac{1}{\sigma_{m}^{2}}\right]
$$

If we set $\frac{\partial^{2} \omega_{m}}{\partial t^{2}}=0$, then $\frac{\left(t-t_{m}\right)^{2}}{\sigma_{m}^{4}}=\frac{1}{\sigma_{m}^{2}}$,so $\sigma_{m}=\left|t-t_{m}\right|$.

For the gaussian function with a single crest, there should be two inflection points, namely $t=t_{m}+\sigma_{m}$ and $t=t_{m}-\sigma_{m}$.If it has $\mathrm{N}$ gaussian peaks, you have $2 \mathrm{~N}$ inflection points. Therefore, by obtaining the first and second partial derivatives of the echo signal, the number and position of the inflection points in the echo signal can be obtained, and the center position, amplitude, half width and other parameters of each gaussian wave peak can be obtained from the parity adjacent inflection points, so as to determine the gaussian expression of the waveform curve.

Set the positions of the two odd-even adjacent inflection points be $t_{2 m-1}$ and $t_{2 m}$. Then the maximum value of the interval $\left[t_{2 m-1}, t_{2 m}\right]$ can be used as the estimated amplitude of the gaussian function, and the position of the maximum value is the mean value of the gaussian wave. The width of gaussian distribution is smaller than that of $\left|t_{2 m-1}-t_{m}\right|$ and $\left|t_{2 m}-t_{m}\right|$, expressed as Eq. (8):

$$
\sigma_{m}=\min \left\{\left|t_{2 m-1}-t_{m}\right|,\left|t_{2 m}-t_{m}\right|\right\}
$$

For GLAS laser height measurement system, an effective ground echo amplitude should be on the dc component of receiving energy. Therefore, only when the value of the gaussian component amplitude determined by the two odd-even adjacent inflection points is greater than that of the echo signal is the effective ground echo. The inflection points caused by noise can be eliminated by determining the minimum gaussian amplitude.

The minimum gaussian amplitude can be determined by background noise threshold and standard deviation as shown in Eq. (9). Then the gaussian peaks with amplitudes less than $A_{\min }$ are removed, leaving only those peaks larger than $A_{\text {min }}$. Meanwhile, these gaussian peaks whose adjacent peak interval is less than the transmitting pulse width will be merged.

$$
A_{\min }=4 \times \sigma+\text { noise }
$$

Where $A_{\min }=$ the minimum gaussian amplitude;

$\sigma=$ the standard deviation of the sampling point;

noise $=$ the estimated mean value of the background noise.

\subsubsection{Fine filtering based on echo data}

When only one wave peak is included in the echo waveform, the geometric model of the waveform can be expressed as:

$$
\omega(t)=A_{t} e^{\frac{-\left(t-t_{m}\right)^{2}}{2 \sigma_{m}^{2}}}+\varepsilon
$$

As shown in Eq. (11), we write the four parameters in matrix form:

$$
c=\left[\varepsilon, A_{t}, t_{m}, \sigma_{m}\right]^{\mathrm{T}}
$$

The following formula can be obtained by taking the partial derivative of the waveform model.

$$
\frac{\partial \omega}{\partial c}=\left[\begin{array}{cccc}
\frac{\partial \omega_{1}}{\partial \varepsilon} & \frac{\partial \omega_{1}}{\partial A_{t}} & \frac{\partial \omega_{1}}{\partial t} & \frac{\partial \omega_{1}}{\partial \sigma} \\
\frac{\partial \omega_{2}}{\partial \varepsilon} & \frac{\partial \omega_{2}}{\partial A_{t}} & \frac{\partial \omega_{2}}{\partial t} & \frac{\partial \omega_{2}}{\partial \sigma} \\
\vdots & \vdots & \vdots & \vdots \\
\frac{\partial \omega_{N}}{\partial \varepsilon} & \frac{\partial \omega_{N}}{\partial A_{t}} & \frac{\partial \omega_{N}}{\partial t} & \frac{\partial \omega_{N}}{\partial \sigma}
\end{array}\right]
$$

Where, $\omega=\left[\omega_{1}, \omega_{1}, \cdots, \omega_{N}\right]$ are the waveform model value corresponding to $\mathrm{N}$ sampling points, and the waveform model is expanded by the first-order Taylor formula:

$$
\omega_{n}^{i+1}=\omega_{n}^{\prime}+\frac{\partial \omega_{n}^{\prime}}{\partial \varepsilon} \delta_{\varepsilon}+\frac{\partial \omega_{n}^{\prime}}{\partial A_{t}} \delta_{A_{t}}+\frac{\partial \omega_{n}^{\prime}}{\partial t} \delta_{t}+\frac{\partial \omega_{n}^{\prime}}{\partial \sigma} \delta_{\sigma}
$$

Where

$$
\begin{aligned}
& \frac{\partial \omega}{\partial \varepsilon}=1 ; \\
& \frac{\partial \omega}{\partial A_{t}}=\exp \left[-\frac{\left(t-t_{m}\right)^{2}}{2 \sigma^{2}}\right] ; \\
& \frac{\partial \omega}{\partial t}=\frac{A_{t}\left(t-t_{m}\right)}{\sigma^{2}} \frac{\partial \omega}{\partial A_{t}} ; \\
& \frac{\partial \omega}{\partial \sigma}=\frac{\left(t-t_{m}\right)}{\sigma} \frac{\partial \omega}{\partial t} .
\end{aligned}
$$

The error between the calculated value of waveform model and the GLAS echo sampling value is as shown in Eq. (14):

$$
l=p-\omega
$$


Let $\frac{\partial \omega}{\partial c}$ be the matrix $\mathrm{A}, A \delta x=l$, then $\delta x=\left[A^{T} A\right]^{-1} A^{T} l$, where $\delta x=\left[\delta_{\varepsilon}, \delta_{A}, \delta_{t}, \delta_{\sigma}\right]^{T}$.

The following formula can be obtained by considering the weights and prior values:

$$
\delta x=\left[A^{T} W A+V_{0}\right]^{-1} A^{T} W l
$$

The new parameters can be obtained:

$$
c^{i+1}=c^{i}+\delta x
$$

The covariance matrix and the variance are shown as Eq.(17) and Eq.(18).

$$
\begin{gathered}
\operatorname{cor}\left(\sigma_{i j}^{2}\right)=\left[A^{T} W A+V_{0}\right]^{-1} \\
s^{2}=\frac{\sum_{n=1}^{N}\left(w_{n}-p_{n}\right)^{2}}{N-4}
\end{gathered}
$$

Based on the refined screening of echo data, echo data whose wave peak number is greater than 1 are eliminated at first, and then waveform fitting is carried out for the single wave peak echo signal. The standard deviation threshold after fitting is set as $5 \mathrm{~ns}$, that is, only the data less than $5 \mathrm{~ns}$ in the single wave peak echo signal is retained for further elimination of waveform data.

\subsubsection{Laser rescreening based on stereo image pair of tie points}

Considering that the acquisition time of the ZY-3 image is not consistent with the acquisition time of the laser elevation point, the terrain may change greatly, so it is necessary to re-screen the stereo image based on the tie point. Since the ZY-3 image is a three-line array stereo image, the object coordinates of the tie points obtained by matching can be obtained through stereo matching and forward intersection.

In this step, we take each GLAS laser point as the center, automatically matched a number of the tie points on the ZY-3 image within a certain range, calculated the three-dimensional coordinates of the tie points, and calculated the elevation residual size of multiple tie points, so as to perform further screening of GLAS laser points.

\section{EXPERIMENT}

\subsection{Experimental Data}

In order to verify the feasibility and reliability of block adjustment with satellite-borne laser altimeter data of ZY-3 image, this paper carried out block adjustment experiments in Shandong province, China. The overall terrain of the experimental area is complex, including plains, hills and mountains, which is conducive to analyzing the distribution of laser point availability under different terrain conditions. In this paper, 112 three linear array stereo images of ZY-3 in Shandong province and 216859 laser points in Shandong province are used as experimental data, and each image data contains a TIFF image of the forward, down, and back view and the corresponding RPB file, with a total data volume of about $270 \mathrm{~GB}$.

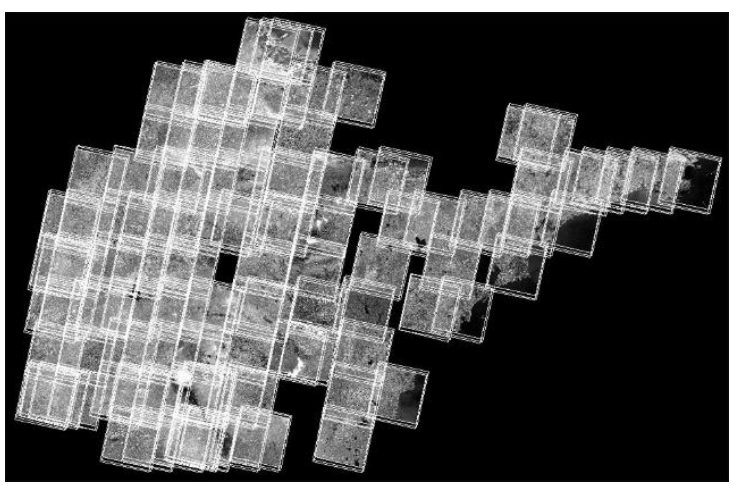

Figure 1. Satellite image distribution map of

Shandong province experimental area resource 3

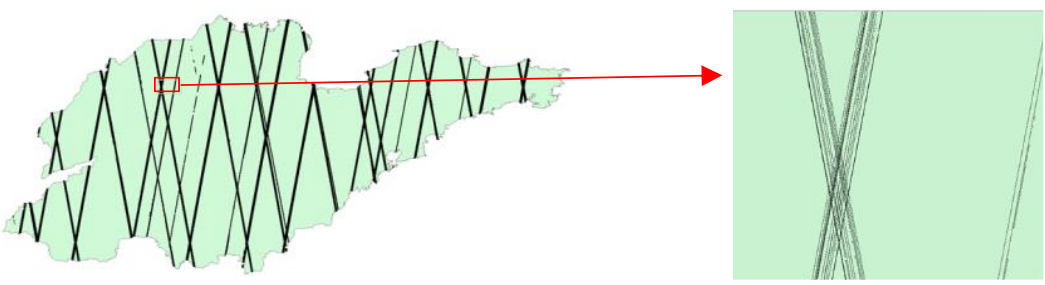

Figure 2. Laser point data distribution and local magnification in the experimental area

In order to verify the change of absolute accuracy of ZY-3 free network adjustment after the introduction of satellite laser altimetry data, and to quantitatively analyze the effectiveness of this method, 911 GPS control points uniformly distributed in Shandong Province are collected as check points to verify the absolute accuracy of regional network adjustment. The plane accuracy of all control points is better than $0.5 \mathrm{~m}$, 
and the elevation accuracy is better than $0.2 \mathrm{~m}$. All control points are points with 3 degree overlap or above, with a maximum of 18 degree overlap. Combined with the record file of control points, the image control coordinates of 911 control points are measured one by one, and the measurement accuracy of image control points is within 1 pixel. All control points are set as check points to quantitatively analyze the absolute accuracy of regional network adjustment.

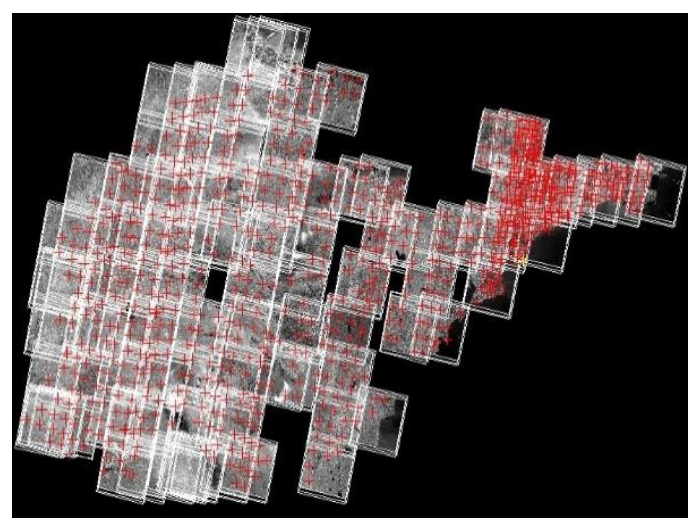

Figure 3. Distribution map of high precision GPS checkpoints in the experimental area

\subsection{Results and Accuracy Analysis}

\subsubsection{Laser spot screening experiment}

According to the fine filtering method based on the echo data, the echo data with the peak number greater than 1 is eliminated first, then the single peak echo signal is fitted with the waveform, and the threshold value of the standard deviation after fitting is set to $5 \mathrm{~ns}$, that is, only the data less than $5 \mathrm{~ns}$ in the fitted single peak echo signal is retained, and the waveform data is further eliminated.

\begin{tabular}{lcc}
\hline & Number of laser points & Data retention \\
\hline Before screening & 216859 & $100 \%$ \\
After screening & 116693 & $53.81 \%$ \\
\hline
\end{tabular}

Table 1. Fine filtering results based on waveform data

Considering that the acquisition time of GLAS data is from 2003 to 2009, and the acquisition time of ZY-3 data is from 2012 to 2014, within this time difference range, there are inevitably some areas with obvious terrain changes, and the real elevation value of these areas on ZY-3 image will be quite different from the elevation value measured by GLAS. Therefore, the laser point re screening based on the stereo image pairs of tie points is carried out.

The number of laser points obtained by precise selection based on echo data is still very large, and many of the data are obtained by repeated orbit in different operation periods. In order to improve the efficiency of filtering based on the tie point image, the data is first diluted. In this paper, a grid of $200 * 200$ pixels size is set up, only one laser point is reserved in each grid, and the points collected in the period of laser2a with better data quality are preferentially reserved, thus reducing the data amount by an order of magnitude. Then, this paper takes the laser point as the center and $50 * 50$ pixels as the range to match the tie points, and removes the laser points with an error of more than $1 \mathrm{~m}$ in the elevation of the matching tie points. The statistical results of the data after the removal are shown in Table 2. The distribution of the final selected reliable laser measuring points is shown in Figure 4.

Number of laser points

Data retention

\begin{tabular}{ccc}
\hline Before screening & 116693 & $100 \%$ \\
Laser spot thinning & 6462 & $5.54 \%$ \\
After the tie point stereo pair screening & 1874 & $1.61 \%$ \\
\hline
\end{tabular}

Table 2. Screening results based on tie points of stereo image pairs 


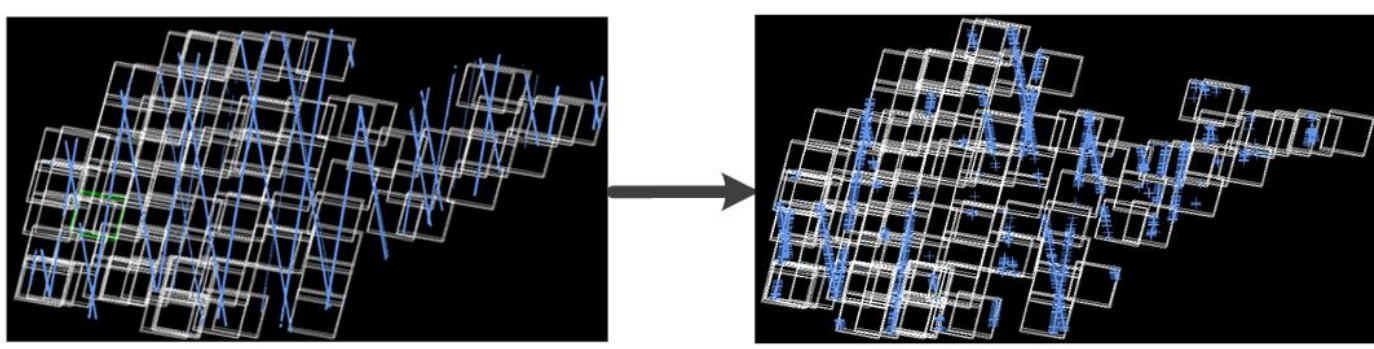

Figure 4. Result map of laser elevation control points in experimental area after screening

It can be seen from the distribution map of laser points after screening that the data retention rate is higher in the west of Shandong Province, while there are more spots deleted in the middle and east of Shandong Province. Combined with the topographic map of Shandong Province, the west part of Shandong Province is the Huang Huai Hai Plain; the middle part is the mountainous area of central Shandong Province, with high and abrupt terrain; the east part is the Shandong Peninsula, mainly composed of hills, with large ups and downs. Therefore, the result of the screening is in line with the expectation, that is, under the condition of 70 meter spot size of GLAS data, only the flat land with small topographic relief can be used as the elevation control point.

\subsubsection{Comparative adjustment experiment in Shandong Province}

The main purpose of this paper is to verify the influence of the selected laser elevation control points on the elevation accuracy of large-scale block adjustment without control points. For this reason, this paper has carried out a comparative experiment of large-scale adjustment without control points in Shandong Province.
In this experiment, the height residual distribution of the check points in the free block adjustment without control and the block adjustment assisted by laser elevation points are compared. In the two groups of comparative experiments, 911 GPS checkpoints are used for accuracy evaluation, and the coordinates of image point measurement of GPS checkpoints are consistent. In the two cases, the elevation residual distribution of checkpoints is shown as Figure 5. In the figure, abscissa represents longitude, unit is degree, ordinate represents latitude, unit is degree, dot represents longitude and latitude coordinate position of check points, arrow in the figure represents the size of inspection point elevation residual value, blue arrow represents inspection point elevation residual value is negative, red arrow head represents elevation residual value is positive. For display convenience, the size value of each arrow is the original elevation residual value divided by 5 , that is, the unit of elevation residual value in the figure is $0.2 \mathrm{~m}$. The residuals of GPS checkpoints of the two groups of experiments are shown in Table 3.

\section{Free net adjustment $\quad$ Laser assisted adjustment}

\begin{tabular}{ccccccc} 
Error term & $\mathbf{X}$ & $\mathbf{Y}$ & $\mathbf{Z}$ & $\mathbf{X}$ & $\mathbf{Y}$ & $\mathbf{Z}$ \\
\cline { 2 - 6 } & & & & & & \\
\hline Mean error & -6.687 & -1.631 & $\mathbf{- 2 . 2 9 7}$ & -6.060 & -1.895 & $\mathbf{0 . 2 1 6}$ \\
Medium error & 7.924 & 4.585 & $\mathbf{3 . 1 9 3}$ & 7.562 & 4.471 & $\mathbf{2 . 0 0 7}$ \\
\hline
\end{tabular}

Table 3. Comparison table of adjustment residuals before and after laser point introduction in experimental area 

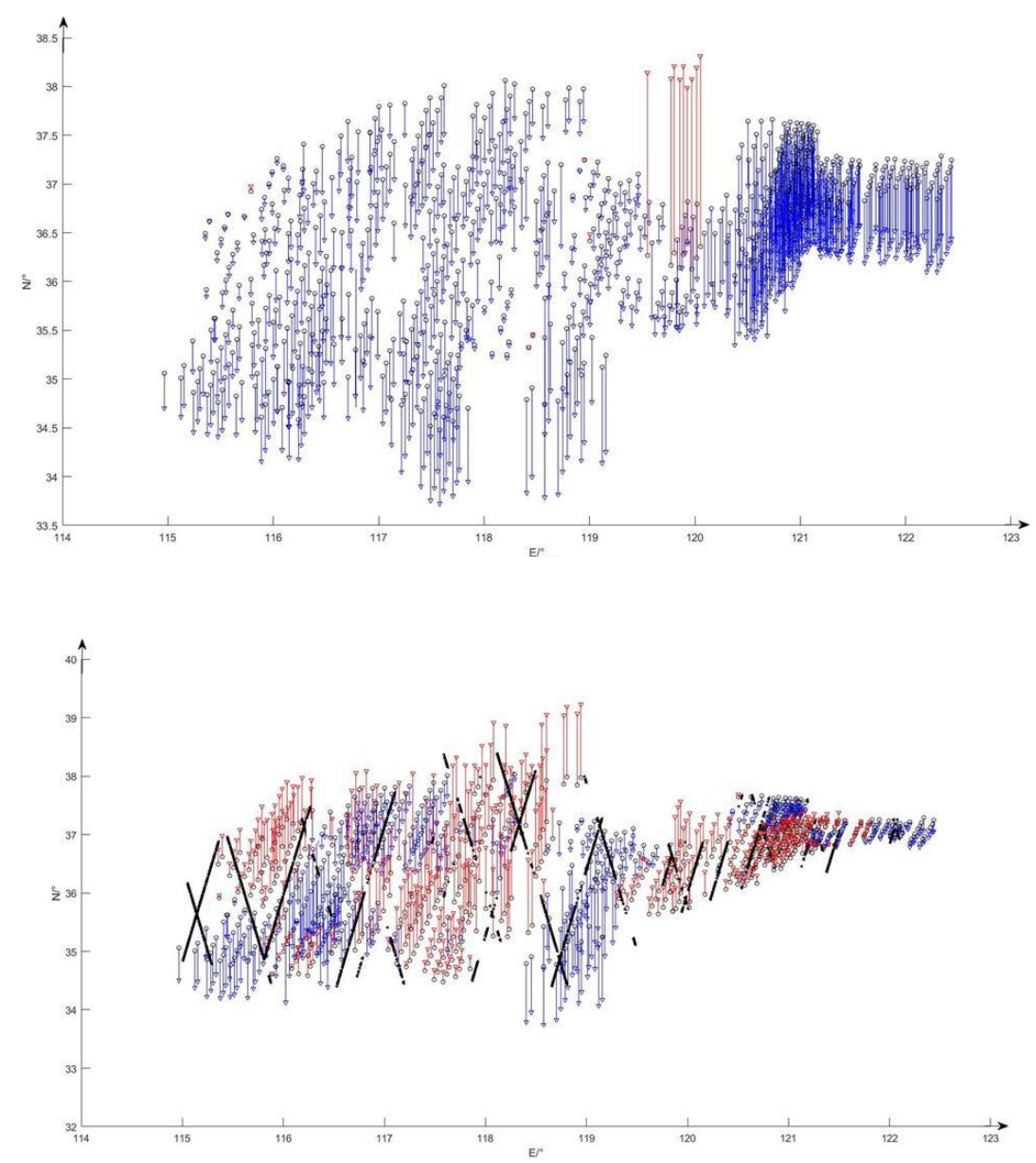

Figure 5. Distribution of elevation residual before and after the introduction of laser point in the experimental area

In Figure 5, the circle indicates the checkpoint, the point indicates the laser point, and the arrow indicates the checkpoint elevation residual value. The upper graph of Figure 5 shows the distribution of the residual height of the free network adjustment. The lower graph shows the regional network adjustment height can after the introduction of laser points.

The experimental results in Shandong Province show that before the introduction of laser point, the mean error of elevation of 911 GPS check points after free network adjustment is -2.297 meters, and the mean error is 3.193 meters. After the introduction of laser point adjustment, the mean error is reduced to 0.216 meters, and the mean error is reduced to 2.00 meters. The system error is basically eliminated, and the mean error is also increased by more than 1 meter.

The above experiments can show that:

(1) Before the introduction of laser point, there are large errors of plane and elevation system in the adjustment of large-scale free network of ZY-3 satellite image under completely uncontrolled conditions. After the introduction of laser point, although the plane system error has not been improved, the elevation system error can be eliminated obviously. The mean error of adjustment elevation is increased from -2.297 to $0.216 \mathrm{~m}$, greatly eliminating the system error; the mean square error of elevation is increased from $3.193 \mathrm{~m}$ to $2.007 \mathrm{~m}$, significantly improving the elevation positioning accuracy of adjustment.

(2) The block adjustment of ZY-3 images with GLAS laser elevation data can eliminate the internal distortion of the regional network to a certain extent. As shown in the experimental results of the large survey area, in the completely uncontrolled condition, the elevation residuals of 911 inspection points are negative except for a few points, and there is a large elevation system error within the regional network. After the introduction of laser point as elevation assistant, the systematic error of this kind of elevation residual can be eliminated basically. the elevation residual of 911 points has positive and negative, and the residual size has a significant decline, which shows that the internal distortion of regional network has been effectively eliminated to some extent.

(3) Through the screening mechanism proposed in this paper, the elevation control points can be screened out with high precision. In the absence of control points, it 
can be used as a good control data in the mapping production of three-dimensional surveying and mapping images.

\section{CONCLUSION}

In this paper, a large-scale block adjustment method with satellite laser altimetry data of ZY-3 image data is proposed. This method first implements a waveform extraction algorithm based on the number of peaks of the full waveform echo data of satellite laser altimetry. Through the analysis of the characteristics of the experimental area and the laser elevation control points, this method first extracts the single back wave peak data, and then performs Gaussian fitting on these data, taking into account the efficiency and accuracy of data extraction. In this paper, we have proposed a laser data filtering mechanism, which is composed of algorithms such as fine screening based on echo data and rescreening based on stereo image pairs of tie points. We have considered various factors that may reduce the accuracy of laser elevation point, such as the topography of laser spot, the time difference between laser spot and ZY-3 image data. In order to verify the reliability of laser altimetry point screening mechanism and the feasibility of non-control block adjustment of ZY-3 image with satellite laser altimetry data, this paper carried out the contrast experiment of large-scale block adjustment of ZY-3 image in Shandong survey area, and quantitatively analyzed the elevation accuracy of free network adjustment and adjustment with laser elevation data through GPS check points obtained from field measurement. The results show that after the introduction of laser elevation data, the mean error of elevation adjustment is increased from $2.2971 \mathrm{~m}$ (absolute value) to 0.2163 , which greatly eliminates the systematic error; the mean error of elevation is increased from $3.1932 \mathrm{~m}$ to $2.007 \mathrm{~m}$, which is more than one meter, thus verifying that the laser altimetry points selected in this paper can meet the elevation accuracy requirements of ZY-3 global mapping and the effectiveness of the adjustment method proposed in this paper.

\section{ACKNOWLEDGEMENTS}

The experiment data and environment are supported by China Center For Resources Satellite Data and Application.

\section{REFERENCES}

Brenner A C, Zwally H J, Bentley C R, et al., 2011: The Algorithm Theoretical Basis Document for the Derivation of Range and Range Distributions from Laser Pulse Waveform Analysis for Surface Elevations, Roughness, Slope, and Vegetation Heights. NASA Technical Reports Server.

Cao, J., Yuan, X., Gong, J., 2015: In-orbit geometric calibration and validation of ZY-3 three-line cameras based on CCD-detector look angles. The Photogrammetric Record, 30(150), 211-226.

Li, D., 1982: On the signal-to-noise ratio in selfcalibrating block adjustment. Journal of Surverying and Mapping, Acta Geodaetica Et Cartographic Sinica, 11(3), 170-184.

Li, R., Deshpande, S., Niu, X., et al., 2008: Geometric Integration of Aerial and High-Resolution Satellite Imagery and Application in Shoreline Mapping. Marine Geodesy, 31(3), 143-159.

Wang, J., Zhang, Y., Zhang, Z.X., et al., 2018: ICESat Laser Points Assisted Block Adjustment for Mapping Satellite-1 Stereo Imagery . Acta Geodaetica et Cartographica Sinica, 47(3), 359-369.

Yang, B., Wang, M., Xu, W., et al., 2017: Large-scale block adjustment without use of ground control points based on the compensation of geometric calibration for ZY-3 images. Isprs Journal of Photogrammetry and Remote Sensing, 1-14.

Zhang, L., Zhang, J.X., Chen, X.Y., et al. 2009: BlockAdjustment with SPOT-5 Imagery and Sparse GCPs Based on RFM. Acta Geodaetica et Cartographica Sinica, 38(4), 302-310.

Zheng, M., Zhang, Y., 2016: DEM-Aided Bundle Adjustment With Multisource Satellite Imagery: ZY-3 and GF-1 in Large Areas. IEEE Geoscience and Remote Sensing Letters, 13(6), 880-884. 